\title{
Shock wave focusing in water inside convergent structures
}

\author{
Chuanxi Wang' and Veronica Eliasson ${ }^{2}$ \\ ${ }^{1}$ Department of Physics and Astronomy, University of Southern California, \\ Los Angeles, CA 90089, USA \\ E-mail: chuanxiw@usc.edu \\ 2Department of Aerospace and Mechanical Engineering, \\ University of Southern California, Los Angeles, CA 90089, USA \\ E-mail: eliasson@usc.edu
}

\begin{abstract}
Experiments on shock focusing in water-filled convergent structures have been performed. A shock wave in water is generated by means of a projectile, launched from a gas gun, which impacts a water-filled convergent structure. Two types of structures have been tested; a bulk material and a thin shell structure. The geometric shape of the convergent structures is given by a logarithmic spiral, and this particular shape is chosen because it maximizes the amount of energy reaching the focal region. High-speed schlieren photography is used to visualize the shock dynamics during the focusing event. Results show that the fluid-structure interaction between the thin shell structure and the shock wave in the water is different from that of a bulk structure; multiple reflections of the shock wave inside the thin shell are reflected back into the water, thus creating a wave train, which is not observed for shock focusing in a bulk material.
\end{abstract}

Keywords: Shock Focusing in Water, Fluid-Structure Interaction, Convergent Structure, Schlieren.

\section{INTRODUCTION}

Underwater explosions (UNDEX) can cause severe damage to nearby marine structures $[1,2]$. A significant portion of the energy generated by UNDEX is carried by the resulting shock wave in water. Thus, knowledge of both shock wave dynamics in water and interaction of the shock wave with water and the structure will facilitate the material selection and design for marine structures subjected to UNDEX.

Previous studies related to fluid-structure interaction and the structural response due to UNDEX has focused on impulsive loading of monolithic plates [3], sandwich beams [4] and composite panels [5]. There are two main distinctions between shock loading on a planar surface and shock loading on a convergent structure. First, when compared to a normal shock impact, a shock focusing event can generate a higher transient pressure in local regions of the fluid, which consequently yields high stress inside the nearby solid structure $[6,7]$. Second, when the shock wave travels into a convergent structure, the interaction time 
between the shock wave and the structure is in general much longer than that of an instantaneous loading, which suggests that more energy can be transferred into the solid structure. These two factors indicate that underwater shock focusing in convergent structures will have complex dynamics and failure modes which are different from the panels subjected to planar shock loading.

Our approach to this type of challenge is to perform laboratory experiments on shock loading of a water-filled convergent structure and monitor the following dynamic response of the structure. The geometric shape of the water-filled convergent structure is chosen to maximize the amount of energy from the incident shock wave reaching the focal region. This particular geometry is given by a so-called logarithmic spiral shape [8, 9]. Further information about the geometry will be given in Section 2 .

In this work, two types of samples have been tested; a bulk material and thin shell structures; see Figure 1 (a) and (b). The bulk material samples are made of polycarbonate and aluminum. Polycarbonate was chosen because it is transparent and thus enables visualization of both longitudinal and transversal waves inside the actual sample. Aluminum was chosen because it is a more realistic material and it has higher wave speeds than polycarbonate. Two types of thin shell structures were tested; low carbon steel of two thicknesses, $6 \mathrm{~mm}$ and 1.29 $\mathrm{mm}$. The coupling between thin shell samples and shock loading in water falls in the moderate coupling regime as suggested by Shepherd et al., [10]. The shell structures are airbacked, i.e. water on one side and air on the other side, to mimic the characteristics of a naval vessel. Previous work on shock focusing in various types of bulk materials have been reported by Eliasson et al., [11]. Results showed a strong coupling between the type of material and the fluid-structure interaction during the shock focusing event.

(a)

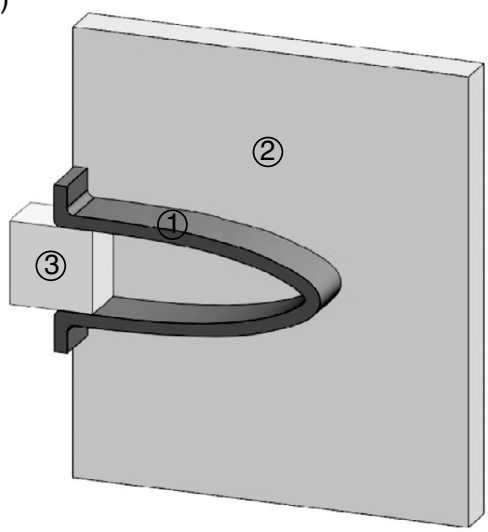

(b)

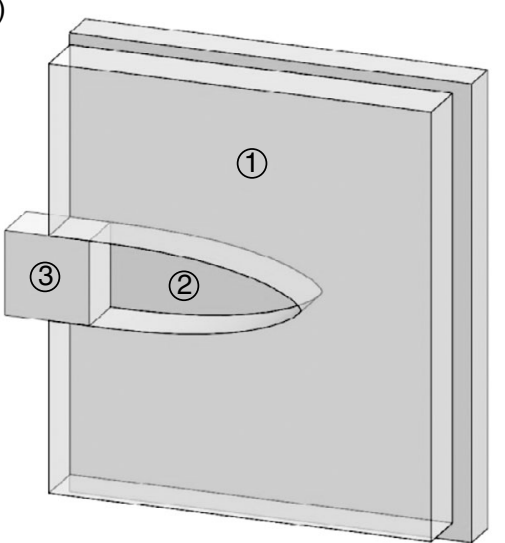

Figure 1 Schematic of the test sample. a1) Air-backed thin shell test material with a water-filled cavity shaped as a logarithmic spiral. a2) Polycarbonate window. Only one of the windows is shown in the figure for clarity. a3) Polycarbonate piston to prevent water leakage. b1) Bulk test material with a water-filled cavity shaped as a logarithmic spiral. b2) Polycarbonate window. Only one of the windows is shown in the figure for clarity. b3) Polycarbonate piston to prevent water leakage. 
To generate the shock wave in water, a gas gun is utilized $[11,12]$, with details explained in section 3.1. The schematic drawing of the experimental laboratory sample is depicted in Figure 1 (a). A thin shell structure in the shape of a logarithmic spiral is mounted between two $12 \mathrm{~mm}$ thick polycarbonate window plates (only one is shown in the figure). The windows are optically transparent for the ease of observation. A polycarbonate blocker is plugged into the opening of the sample, for the purpose of both sealing water inside and transmitting the shock wave into water. All the contacts between different parts are wellsealed using silicone glue or epoxy to prevent water leakage. Once the shock wave enters the region of interest, a visualization technique called schlieren optics is used to unveil the change of refractive index, or equivalently the density change, in the water, see details in Section 3.3. The visualization results can be used to identify the dynamics of the coupled system. Post-test analysis is also performed to quantify the damage to the samples.

\section{LOGARITHMIC SPIRAL SHAPE}

Shock focusing in a logarithmic spiral shape is being investigated due to the ability of a logarithmic spiral to minimize reflections off the surrounding boundary. A logarithmic spiral is depicted in Figure 2.

The derivation of the logarithmic spiral is based on Whitham's ray-shock theory, or socalled Geometrical Shock Dynamics (GSD) theory $[8,13]$. The form of the curve can be expressed as following

$$
r=\frac{L}{\cos \chi} e^{\frac{\chi-\theta}{\tan \theta}},
$$

where the variables $r, L, \chi$ and $\theta$ are shown in Figure 2. Previous work using both simulations and experiments have shown that a two-dimensional logarithmic spiral geometry produces shock focusing in air without observable reflections off the surrounding boundary $[9,14]$.

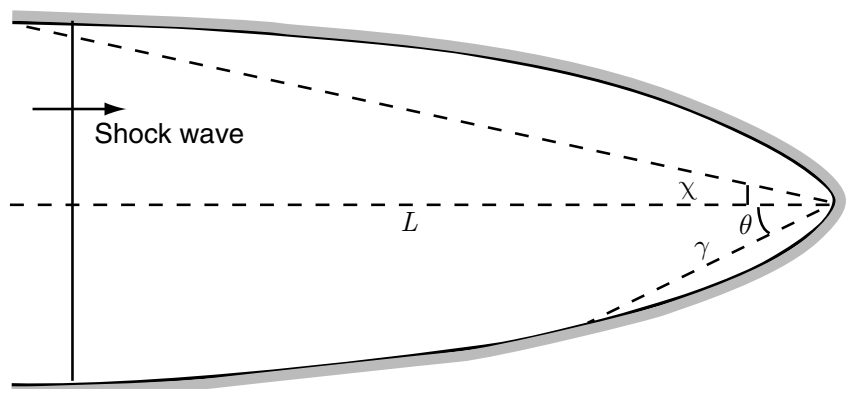

Figure $2 L$ is the length of the logarithmic spiral duct. The angle $\chi$ determines the overall shape and also the local curvature. $\theta$ and $r$ are polar coordinates with the origin at the tip of the duct. 
Here, an extension of the GSD theory is presented for using water as shock medium instead of air, as previously derived, [13]. In order to account for water instead of air, the GSD theory has to be modified by changing the equation of state (EOS) of the shock focusing medium from an ideal gas EOS to an EOS valid for water. Here, a stiffened EOS, also referred to as Tammann-Tait EOS [15], has been used. The stiffened EOS is given by

$$
e=\frac{p+\gamma_{\infty} p_{\infty}}{\rho\left(\gamma_{\infty}-1\right)}
$$

The variable $e$ is the internal energy, $p$ is the pressure, $\rho$ is the density; and the constants $\gamma_{\infty}=7.415, p_{\infty}=2.962 \times 10^{8} \mathrm{~Pa}$ are chosen to match the speed of sound of water, see Eliasson [11]. The stiffened EOS has been used in previous works related to shock waves in water, such as underwater explosions [16], and the collapse of shock-induced bubbles in water [17].

Rewriting the Rankine-Hugoniot jump conditions using the stiffened EOS gives the following equations

$$
\begin{aligned}
& u_{2}=a_{1} \frac{2\left(M^{2}-1\right)}{\left(\gamma_{\infty}+1\right) M}, \\
& \rho_{2}=\rho_{1} \frac{\left(\gamma_{\infty}+1\right) M^{2}}{\left(\gamma_{\infty}-1\right) M^{2}+2}, \\
& p_{2}=\rho_{1} a_{1}^{2} \frac{2\left(M^{2}-1\right)}{\gamma_{\infty}+1}+p_{1} .
\end{aligned}
$$

Here, $M$, is the shock Mach number, $u$, is the particle velocity, and $a$ is the speed of sound. Quantities with a subscript 1 denote variables in the undisturbed region ahead of the shock wave, and quantities with a subscript 2 denote variables in the region behind the shock. This convention will remain the same through this paper. The RankineHugoniot conditions, Eqns (3) - (5), can now be substituted into the following equation for the $C+$ characteristic,

$$
\frac{d p_{2}}{d x}+a_{2} \rho_{2} \frac{d u_{2}}{d x}+\frac{\rho_{2} a_{2}^{2} u_{2}}{u_{2}+a_{2}} \frac{1}{A} \frac{d A}{d x}=0
$$

where $A=A(x)$ denotes the cross-sectional area of a ray tube where the $C+$ characteristic is being considered. The $C+$ characteristic belongs to the family of characteristics having positive slope on the time-position graph, when considering flow properties behind a shock wave moving in a quasi one-dimensional tube. By substituting Eqns (3) - (5) into Eqn (6), the result is 


$$
\frac{M}{M^{2}-1} \lambda(M) \frac{d M}{d x}+\frac{1}{A} \frac{d A}{d x}=0
$$

where

$$
\begin{aligned}
& \lambda(M)=\left(1+\frac{2}{\gamma_{\infty}+1} \frac{1-\mu^{2}}{\mu}\right)\left(1+2 \mu+\frac{1}{M}\right), \\
& \mu^{2}=\frac{\left(\gamma_{\infty}-1\right) M^{2}+2}{2 \gamma_{\infty} M^{2}-\left(\gamma_{\infty}-1\right)} .
\end{aligned}
$$

Eqn (7) can be rewritten in the form

$$
\frac{A}{M(d A / d M)}=-\frac{M^{2}-1}{\lambda(M) M^{2}} .
$$

Now, by using Whitham's GSD theory, the characteristic angle is given by

$$
\tan \chi=\left(-\frac{A}{M(d A / d M)}\right)^{\frac{1}{2}}
$$

Finally, Eqn (11) can be rewritten in terms of the shock Mach number $M$, and $\gamma_{\infty}$, the parameter from the stiffened EOS,

$$
\tan \chi=\left(\frac{M^{2}-1}{\lambda(M) M^{2}}\right)^{\frac{1}{2}} .
$$

where $\lambda(M)$ is given by Eqn (8). With $\chi$ known, we are able to predict the shape of a logarithmic spiral in the case of shock wave focusing in water.

As seen in Eqn (12), the characteristic angle $\chi$ depends only on the Mach number of the shock wave and $\gamma_{\infty}$ from the equation of state. However, the Mach number depends largely on the nature of the problem under study. In this paper, we focus on the impact of shock waves from UNDEX. The value of the incident shock wave is determined considering a 135kilogram Trinitrotoluene (TNT) charge. The peak pressure behind a spherical shock wave is decreasing with time and distance away from the source. Thus, the shock front will experience a deceleration, and the Mach number of the shock wave will decay from $M=1.2$ at the close proximity of the charge, to $M=1$, at a distance far away [18]. Since we are considering structures close to where UNDEX happens, we chose an initial Mach number of $M=1.1$ for all the experiments. This Mach number represents a shock wave 3 meters away from a 135-kilogram TNT charge. 


\section{EXPERIMENTAL METHOD}

This section on experimental methods is divided into three parts. The first part contains a brief description of the impact method and how the velocity of the projectile is controlled [11]. In the second part, the visualization technique and the triggering system is explained for using both high-resolution and high-speed photography. In the third part, the procedures of making composite samples are listed.

\subsection{GAS GUN OPERATION AND SHOCK GENERATION}

The gas gun shown in Figure 3 is a single stage gas gun. The gun barrel is a $2.1 \mathrm{~m}$ long steel tube with $50.8 \mathrm{~mm}$ inner diameter. The gun barrel is connected to a pressure chamber filled with compressed air. The projectile is a $75 \mathrm{~mm}$ long cylinder with $50 \mathrm{~mm}$ diameter made out of PTFE-filled Delrin. During the experiment, the chamber pressure of the gas gun is measured using a digital pressure gauge (Druck DPI 104, absolute type, $6.9 \times 10^{5} \mathrm{~Pa}(100$ psi)). The velocity of the projectile is measured using two optical sensors (Avago Technology HFBR-1505CZ), which are placed close to the exit of the gun barrel. The optical sensor receives light from a light emitting diode (LED). When the moving projectile blocks the light from the LED, a signal is generated. By this means, two optical sensors placed a known distance apart will give the velocity of the projectile. The velocity can also be measured by looking at the signal from one LED, and measuring the time when the projectile blocks the light for that particular sensor. This technique gives us three different velocity measurements during the same run. For all the experiments, the pressure chamber is operated at $2.75 \times 10^{5}$ $\mathrm{Pa}$ (40 psi), which corresponds to a projectile velocity of $48.0 \pm 0.1 \mathrm{~m} / \mathrm{s}$ at the barrel exit. The projectile will then impact onto the blocker that seals the sample, see Figure 1, which will then deliver an energetic shock wave into the water section.

\subsection{WAVE PROPAGATION}

For the following discussion we will use subscript $p$ for the projectile, $b$ for the blocker, $w$ for water, $p b$ for the projectile-blocker interface and $b w$ for the blocker-water interface, see more in [19].

The moving projectile will strike normally onto the polycarbonate blocker, resulting in a weak shock wave being transmitted into the water. A detailed $x-t$ diagram of the incident

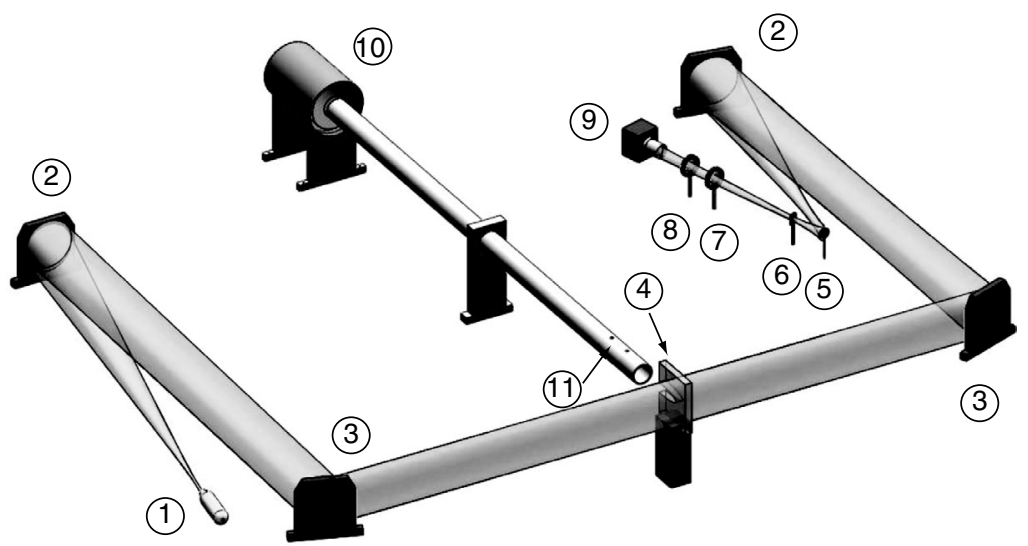

Figure 3 Schematic of the experimental setup. 1) Spark source. 2) $254 \mathrm{~mm}$ diameter concave mirror. 3) $254 \mathrm{~mm}$ diameter flat mirror. 4) Test section. 5) 25.4 $\mathrm{mm}$ diameter flat mirror. 6) Knife edge. 7) 300 mm lens. 8) $200 \mathrm{~mm}$ lens. 9) Camera. 10) Gas gun. 11) Velocity sensors. 
and reflected waves is shown in Figure 4. The projectile is moving from left to right in the figure. At time $t_{1}$, the projectile impacts onto the polycarbonate blocker which is initially is at rest. The impact generates a left-going shock wave in the projectile and a right-going shock wave in the blocker. Physical properties related to wave transmission and reflection are listed in Table 1, [20, 21]:

The shock wave in the projectile propagates with a lower velocity than that in the blocker, which is indicated by a steeper slope on the $\mathrm{x}$-t diagram. Once the shock wave in the blocker

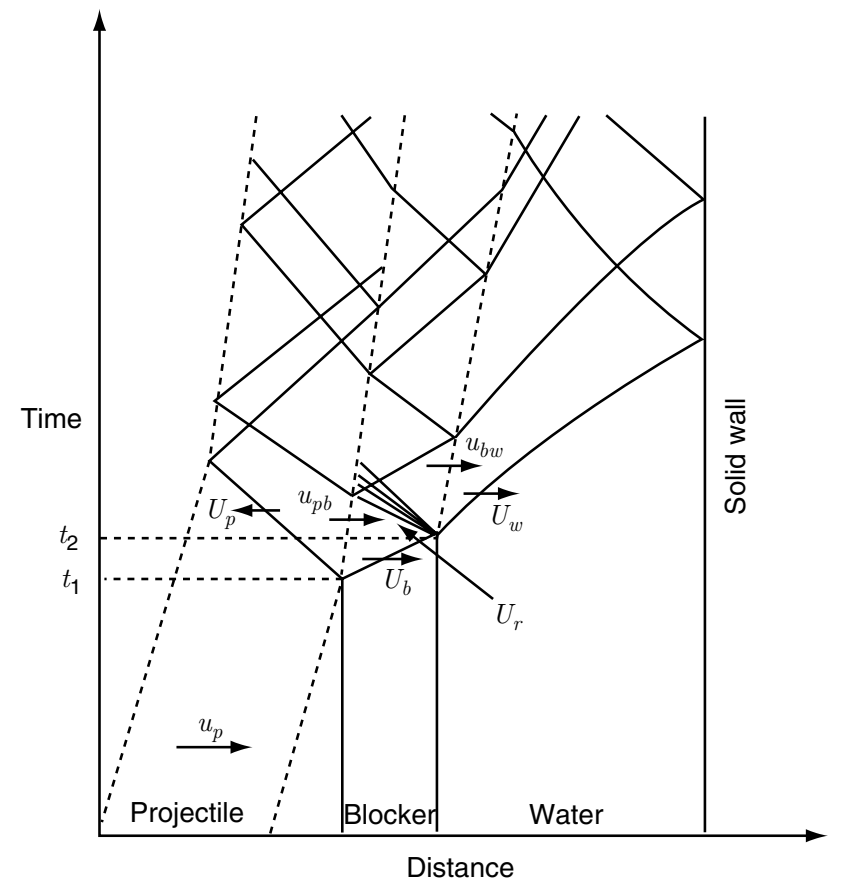

Figure 4 An x-t graph of incident and reflected waves for a polycarbonate sample. $u_{p}, u_{p b}$ and $u_{b w}$ are the velocities of the projectile, the particle velocity at the projectile-blocker interface and the particle velocity at the blocker-water interface. $U_{p}, U_{b}$ and $U_{w}$ are the shock wave velocities in the projectile, polycarbonate blocker and water, respectively. $U_{r}$ is the velocity of the reflected rarefaction wave front in the blocker.

Table 1 Material properties, $[20,21] .^{\star \star}$ For Delrin, $C_{0}$ is picked to be the same as the speed of sound and $S$ is set to be 0 .

Delrin

Polycarbonate

Water

\begin{tabular}{lccc}
\hline$\rho \frac{k g}{\left[m^{3}\right]}$ & 1420 & 1210 & 1000 \\
\hline$a \frac{m}{[s]}$ & 1531 & 2270 & 1482 \\
\hline$C_{0} \frac{m}{[s]}$ & $1531^{* *}$ & 2330 & 1450 \\
\hline$S$ & $0^{* *}$ & 1.57 & 1.33 \\
\hline
\end{tabular}


is formed, it will propagate to the right until reaching the interface between the block and the water domain at time $t_{2}$. The behavior of the shock wave at the interface depends on the impedance mismatch between the media on both sides. The impedance of polycarbonate (subscript $p c$ ) and water (subscript $w$ ) are given by

$$
\begin{aligned}
& Z_{p c}=a_{p c} \rho_{p c}=2.7 \times 10^{6} \mathrm{~kg} / \mathrm{m}^{2} \mathrm{~s}, \\
& Z_{w}=a_{w} \rho_{w}=1.5 \times 10^{6} \mathrm{~kg} / \mathrm{m}^{2} \mathrm{~s} .
\end{aligned}
$$

Because the impedance in the polycarbonate blocker is larger than that of the water, $Z_{p c}>Z_{w}$, a shock wave will be transmitted into the water. At the same time, a rarefaction wave will be reflected back because the blocker-water interface is moving faster than the projectile-blocker interface to the right, resulting in an expansion of the block. The change in curvature of the shock wave path on the $x-t$ diagram indicates that in general the shock wave experiences acceleration when moving toward the tip of the converging structure.

Pressure and particle velocity, $P-u$, Hugoniot equations are utilized [19] in order to quantitatively determine the speed of the resulting shock wave in the water, see Figure 5. At the projectile-blocker interface, the pressure $p_{1}$ and the particle velocity $u_{1}$ on each side has to match each other to maintain a contact interface. The velocity of the left-going shock wave in projectile is described by

$$
U_{p}=C_{p}+S_{p}\left(u_{1}-u_{p}\right)
$$

in which, $U_{p}$ and $u_{p}$ are the shock velocity and the projectile velocity; $C_{p}$ and $S_{p}$ are $C_{0}$ and $S$ respectively in Table 1 for the projectile material. Because $C_{p}$ and $S_{p}$ for PTFE-filled

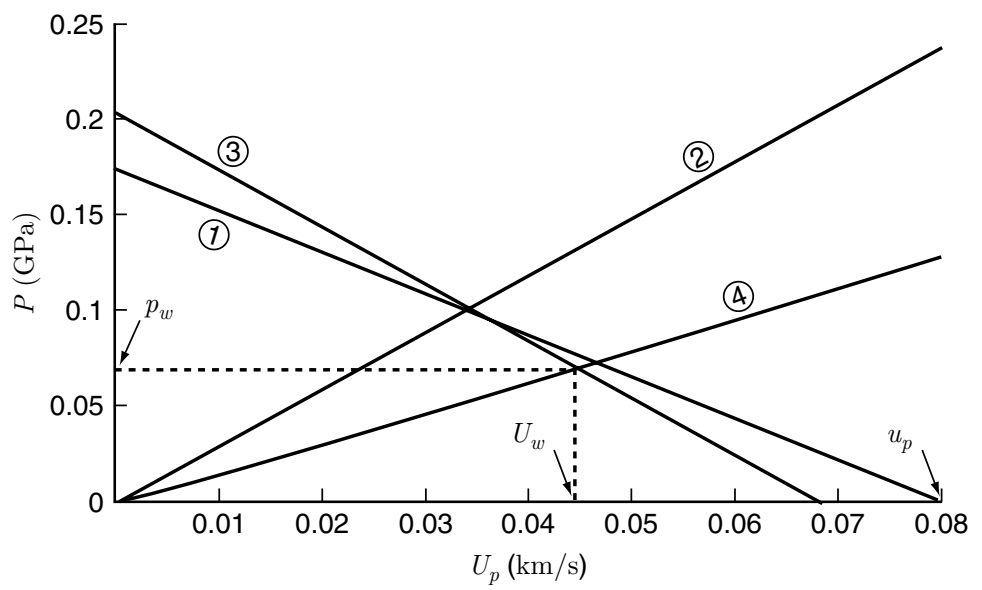

Figure 5 Material properties. Hugoniot equations on $P-u$ graph. 1) Left-going shock wave in the projectile. 2) Right-going shock wave in the blocker. 3) Leftgoing rarefaction wave in the blocker. 4) Right-going shock wave in the water. 
Delrin are not readily available in the literature, and the experiments performed are essentially in a low-speed regime, $U_{p}$ is close to the sound speed, $a_{p}$. Thus, $U_{p}=a_{p}$ is a reasonable approximation in our experiments. The hydrodynamic pressure of the projectile material behind the shock wave $p_{p}$ is given by

$$
p_{p}=\rho_{p} a_{p}\left(u_{1}-u_{p}\right)
$$

For the right-going shock wave in blocker, the hydrodynamic pressure, $p_{b}$, is

$$
p_{b}=\rho_{b} u_{1}\left(C_{b}+S_{b} u_{1}\right)
$$

Since the hydrodynamic pressures are the same at the interface, $p_{p}=p_{b}$, Eqns (16) and (17) can be solved with only two unknown variables. The same technique can be applied to the blocker-water interface to get the particle velocity $u_{2}$ in the water. Once $u_{2}$ is obtained, the following equation

$$
U_{w}=C_{w}+S_{w} u_{2}
$$

can be used to determine the shock velocity in water. This procedure enables a systematic way to relate the impact velocity of the projectile and the resulting shock velocity in the water, as plotted in Figure 6.

\subsection{VISUALIZATION TECHNIQUE}

A Z-folded schlieren optical system has been set up in the laboratory, (see [22] for a detailed explanation of the principles of schlieren techniques). The schlieren system consists of a

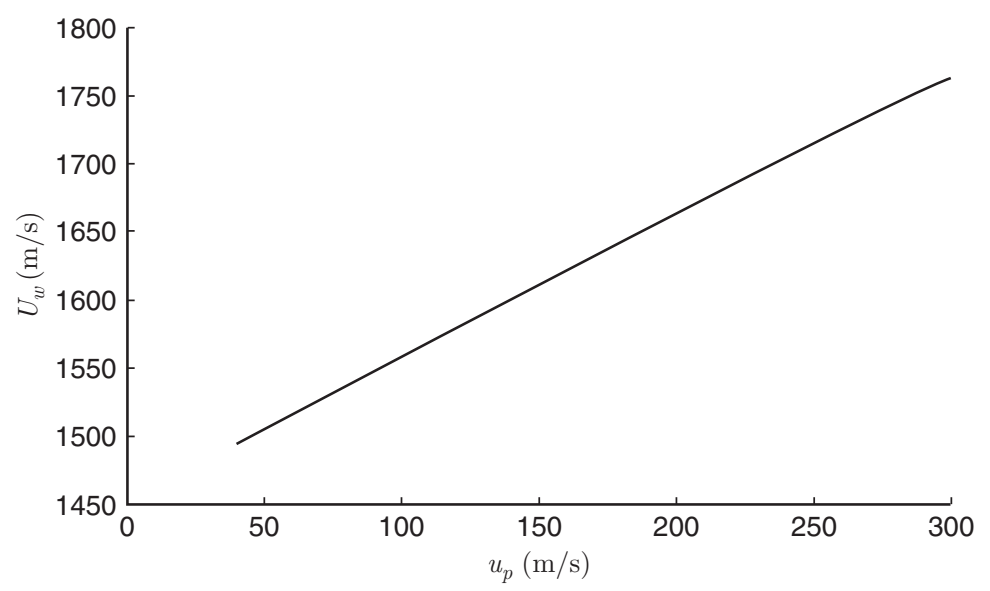

Figure 6 Shock wave velocity in the water as a function of the projectile impact velocity. 
light source, two $254 \mathrm{~mm}$ diameter concave mirrors, two $254 \mathrm{~mm}$ diameter flat mirrors, a knife edge and a Nikon D90 SLR camera for high-resolution single-image photography, or a high-speed video camera, see Figure 3. The corresponding viewing size of the test section is $150 \mathrm{~mm} \times 256 \mathrm{~mm}$. For high-resolution photography experiments (using the Nikon camera), the camera shutter opens for 2 seconds waiting for a flash from the spark light source. When the impact between the projectile and the blocker takes place, a control signal is sent to a pulse/delay generator (BNC Model 575). The delay generator receives the signal and outputs a TTL signal to the light source with a pre-determined delay time. The time delay takes into account the time needed for the shock wave to travel from the projectile/blocker surface into the water and reach the location of interest inside the waterfilled cavity. The light from the spark source passes through the test section and is recorded by the camera. When using high-speed photography, the high-speed camera records a series of pictures with lower resolution, $1360 \times 1024$ pixels, using a preset internal framing rate.

\section{RESULTS AND DISCUSSION}

Using a SIMD16 camera (Specialised Imaging, Inc.), 16 frames of high-speed images were recorded for the $6 \mathrm{~mm}$ thick steel sample, as shown in Figure 7. Because the impact was not

(a)

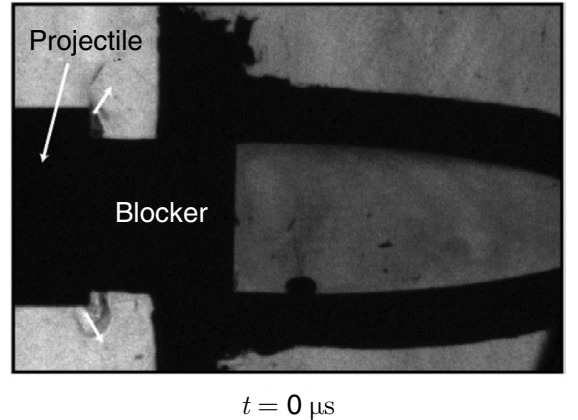

(c)

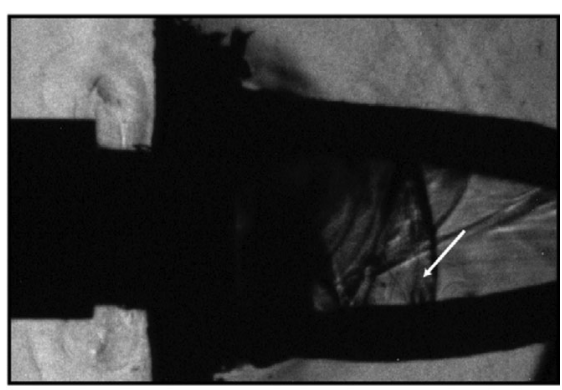

$t=36 \mu \mathrm{s}$ (b)

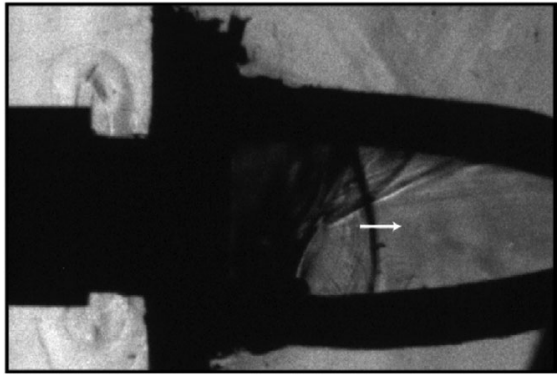

$t=27 \mu \mathrm{s}$

(d)

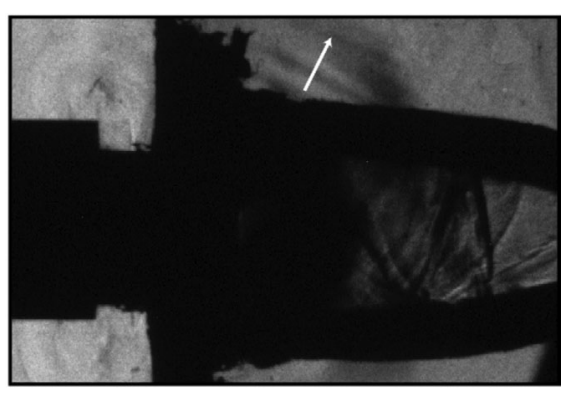

$t=45 \mu \mathrm{s}$

Figure 7 A sequence of schlieren images for the $6 \mathrm{~mm}$ steel sample recorded with a high-speed camera. Impact speed $u=48 \pm 0.1 \mathrm{~m} / \mathrm{s}$. Exposure time $50 \mathrm{~ns}$. (a) The white arrow indicates the propagation of shock waves in air from the impact location. (b) The white arrow indicates the incoming shock wave front. (c) The wave train pattern is pointed out by a white arrow. (d) Acoustic waves get transmitted into air after the passage of the shock front. 
planar, the shock wave enters the cavity at an angle rather than perpendicular. However, some basic features of this type of sample can still be obtained. At $t=0 \mu \mathrm{s}$, the projectile has impacted onto the piston, which is suggested by the toroidal shaped shock generated by the collision. The shock wave in the air is indicated by arrows. At $t=36 \mu \mathrm{s}$, the shock wave enters the water and moves to the center of the view. At $t=27 \mu \mathrm{s}$, multiple reflection patterns appear at the watersolid interface, as pointed out by a white arrow. Pressure waves will reflect inside the shell due to the finite thickness of the sample. Every time a wave returns to the water/shell interface, it will transmit a pressure wave into the water, thus forming this wave train pattern behind the shock front. A direct measurement of the separation between the patterns on the picture confirms this mechanism. The separation or the wavelength of the wave train is $2.58 \pm 0.21 \mathrm{~mm}$, which corresponds to the distance the shock front has traveled when a secondary reflection is transmitted back to the water domain. The time duration can be calculated by

$$
t_{s}=\frac{\lambda}{a}
$$

in which, $\lambda$ is the wavelength of the wave train, and $a$ is the speed of sound in water $\left(1480 \mathrm{~m} / \mathrm{s}\right.$ for room temperature). The time, $t_{s}$, obtained is $1.74 \pm 0.14 \mu \mathrm{s}$, which is the same as the time needed for the pressure wave to travel through the entire thickness of the steel shell and reflect back to the water domain. At later times, the coupling between the air and the test sample is confirmed by observing waves in the air, see e.g. the arrow pointing at the shocks in air in Figure 7(d).

Shock focusing using the thin sample is shown in Figure 8 (e) and (f). As seen in Figure 8 (e) and (f) a distinct wave structure appears behind the incident shock wave. The wave structure for the thinner steel sample is similar to the pattern observed for the thicker steel sample, when comparing Figure 7 (c) with Figure 8 (f). There are two major differences; (i) The location of the wave train; for the thinner sample the location of the wave train is in the center of the water-filled cavity, while it is along the edges of the logarithmic spiral for the thicker sample. (ii) The wavelength of the pattern suggests a different cause for its origin. As explained before, the wave pattern in the thick sample is due to multiple reflections in the shell walls. If we apply the same argument for the thinner sample, the expected wavelength of the wave train pattern should be less than the one for the thick sample. However, when measured from the photograph, the wavelength for the $1.29 \mathrm{~mm}$ thick steel sample is $3.55 \pm 0.21 \mathrm{~mm}$, which is greater than that of the $6 \mathrm{~mm}$ thick steel sample. The reason is that when the thickness of the shell is reduced, significant flexural waves will be induced in the shell as a result of the shock loading. This behavior has been studied further by Shepherd and Inaba, [10], for applications of shock loading in tubular structures. In water, evanescent acoustic waves can be generated as a result of the flexural wave induced in the surrounding solid [23]. We believe that the wave pattern observed in the thin sample is caused by the flexural waves in the thin shell.

In Figure 8 (a) and (c), visualization results for the polycarbonate bulk sample and the aluminum bulk sample are shown. The reflection of the shock wave is stronger for the aluminum sample than that for the polycarbonate sample, due to a greater impedance mismatch between solid aluminum and water. A major difference of the shock dynamics in water for the bulk surrounding materials as when compared to the shell steel structures is that there is no wave train behind the incident shock wave. This is because the coupling between the bulk material and water is weak. 
(a)

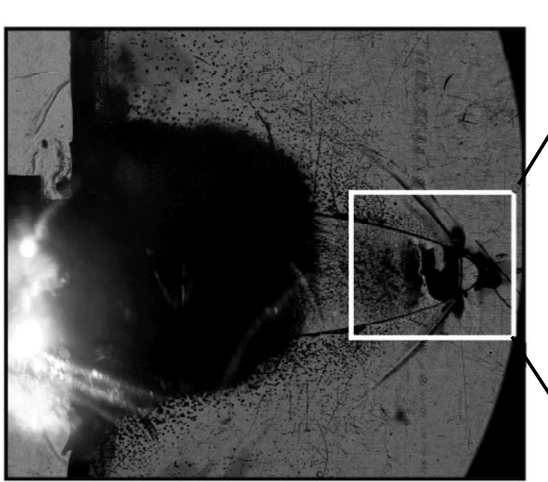

(b)

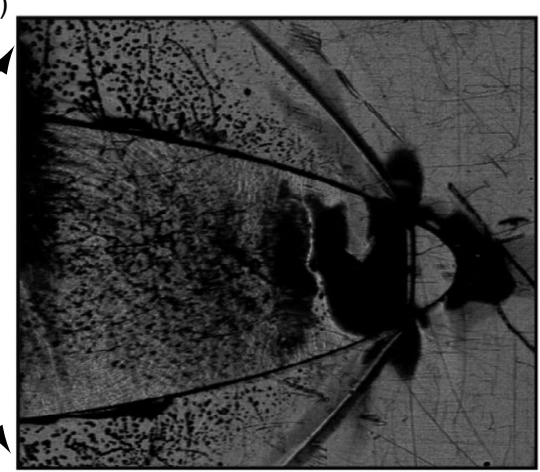

(c)

(d)

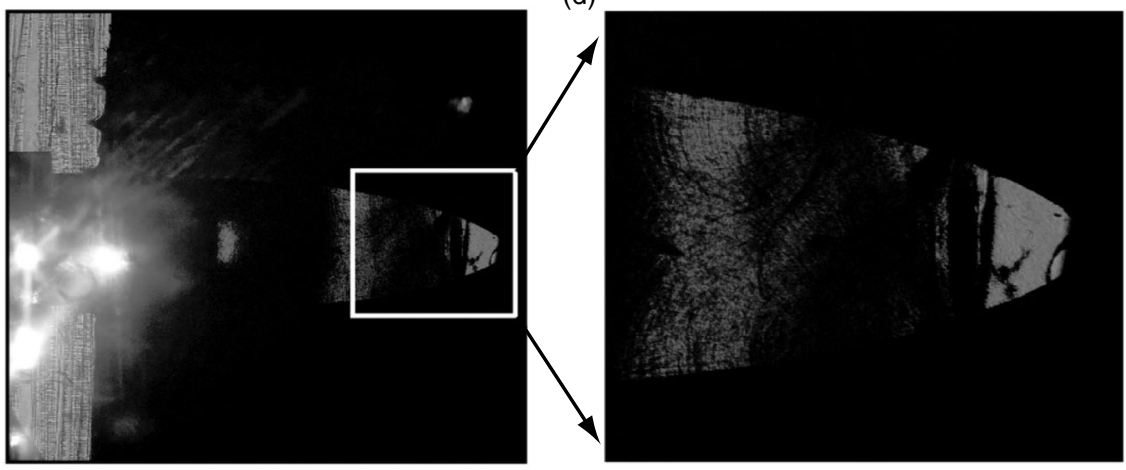

(e)

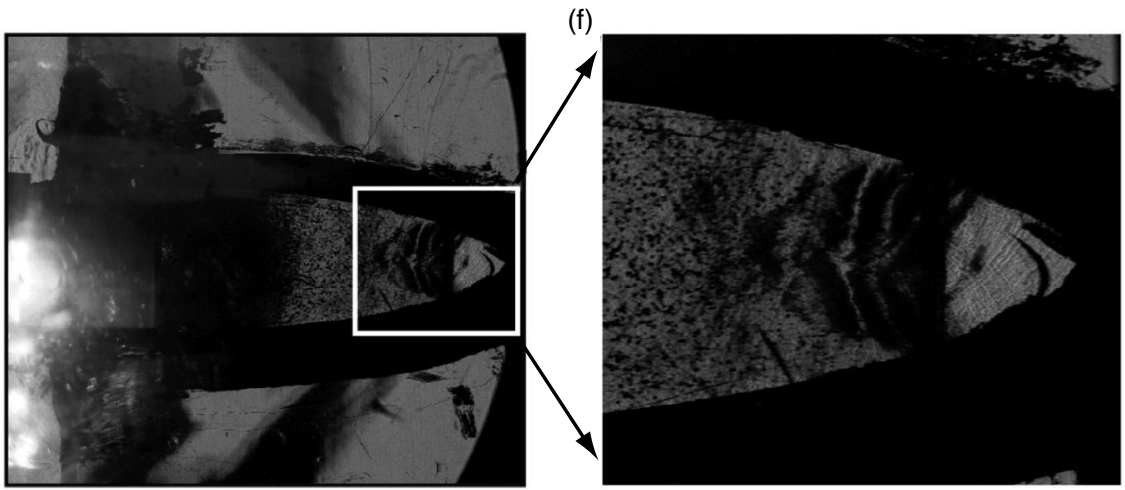

Figure 8 Comparison between $1.29 \mathrm{~mm}$ thick steel and the bulk surrounding materials. (a) Bulk polycarbonate sample. (b) Enlargement of the shock front in (a). (c) Bulk aluminum sample. (d) Enlarged view of the shock front in (c). (e) $1.29 \mathrm{~mm}$ thick steel. (f) Enlargement of the shock front in (e).

\section{PRELIMINARY TESTS FOR CARBON FIBER SHELLS}

Lightweight composite materials, such as carbon fiber composites, are being used increasingly for both military and civilian applications, in light of their high strength-toweight ratio, corrosion resistance etc. [24, 25]. Experimental [26] and numerical [27] investigations have been conducted on numerous types of composite materials regarding the material strength and failure modes under static or dynamic test conditions [28, 29]. Here, 


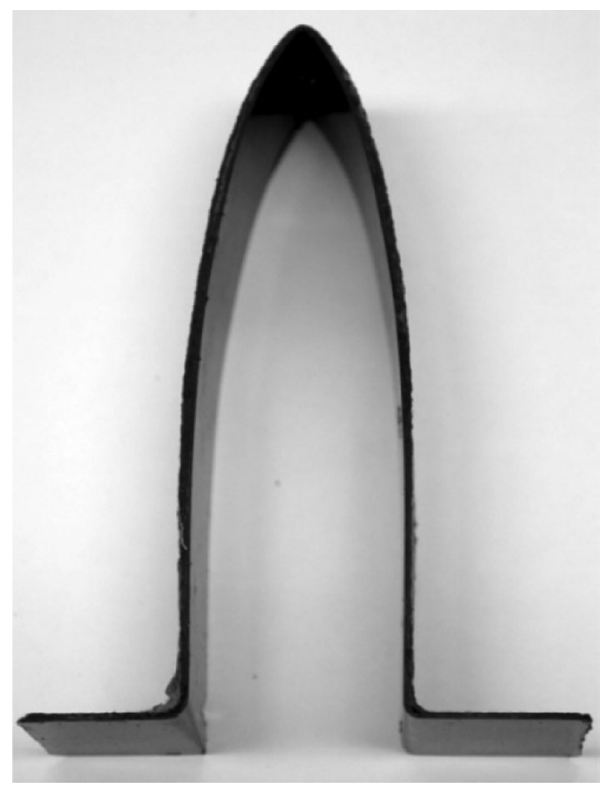

Figure 9 Carbon fiber composite test sample.

preliminary tests using a $2 \mathrm{~mm}$ thick carbon fiber sample have been performed, Figure 9 . The carbon fiber sample has the same shape, a logarithmic spiral, as the rest of the samples in this study. After shock impact, the sample typically breaks at the tip. Post-analysis using a Scanning Electron Microscope (SEM) shows the nature of the damage, see Figure 10. It is clear that the pressure increase from the shock wave inside the water is pushing the sample apart. From the SEM images, the inner layer of the tip shows signs of stretching failure, while the outer layer shows compression patterns.

\section{CONCLUSIONS}

In this current study, a special geometric shape is utilized to focus the shock wave in water, in order to have a maximum transient pressure at the focal region. Effects from the surrounding materials to this focusing process are visualized by using schlieren technique. The interactions between the shock wave and the surrounding solid structure are distinguished between bulk type samples and thin shell type samples. Unique wave train patterns are observed in the thin shell type samples regardless their thickness, which is in general absent in the bulk type samples. However, different mechanisms for the wave patterns are explained and speculated by the authors. The thickness of the test sample plays an important role when considering the fluid structure coupling. The preliminary tests using carbon fiber samples show that the sample breaks at the tip region. This is a different failure mode when compared to the previously mentioned steel structures. The steel structures deform but do not break.

In the future, further information about the flexural waves in solid will be available by applying strain gauge measurement. A quantitative schlieren technique is developed for obtaining density distribution inside the water domain. High-speed visualizations of the carbon fiber structures will be used to show how the shock wave interacts with the surrounding structure. In addition, nondestructive computer tomography will be utilized to obtain information inside the samples before they fail. 
(a)

(b)

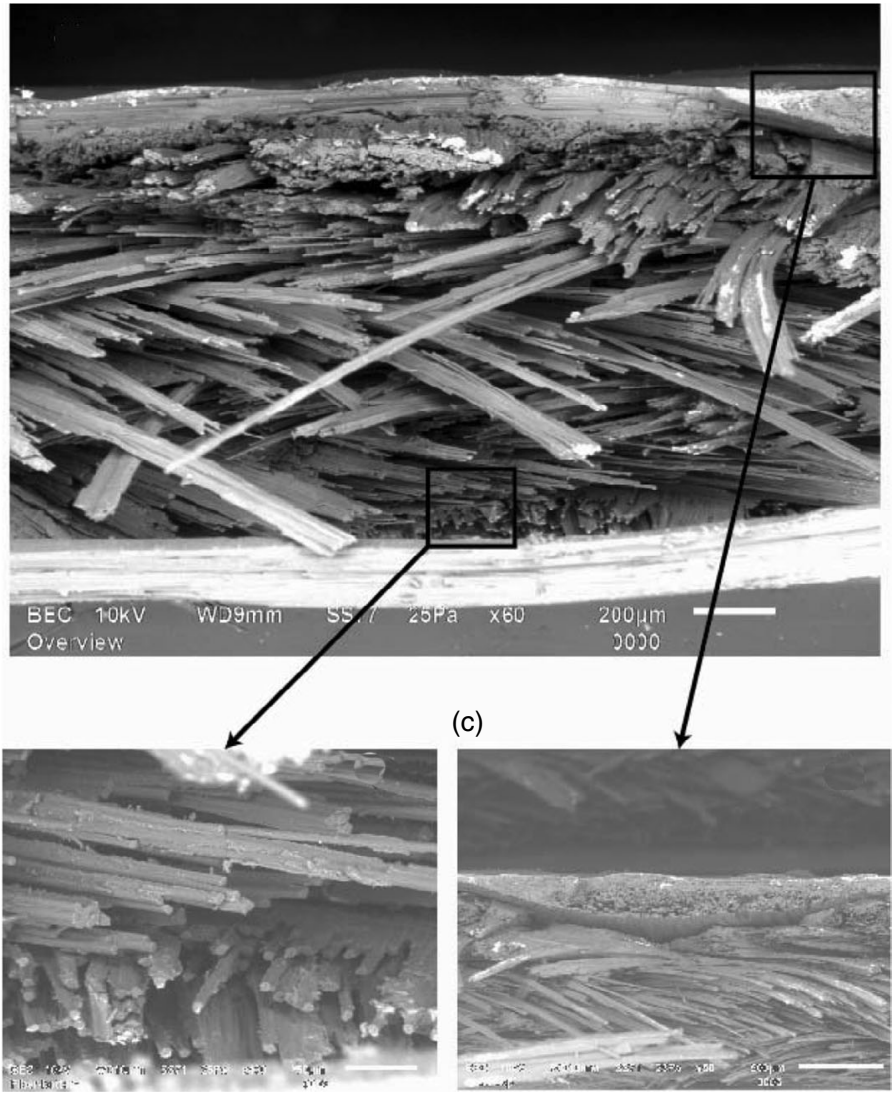

Figure 10 SEM images of the damaged carbon fiber sample. (a) Overview of the failure cross section. (b) Region in the sample close to the inner surface which is in contact with water. (c) Region close to the outer surface.

\section{ACKNOWLEDGEMENT}

This work is supported by Office of Naval Research through a MURI Grant Number N00014-06-1-0730 (Dr. Y.D.S. Rajapakse, Program Manager). The authors wish to thank Dr. Young Kwon for his invitation for this special issue. The authors also want to thank the USC machine shop and the Caltech Aero shop, Ms. Lessa Grunenfelder and Dr. Steven Nutt for preparing the composite samples, and Ms. Catherina Ticsay for helping with Figure 3.

\section{REFERENCES}

[1] Swisdak, M.M., Explosion Effects and Properties: Part II - Explosion Effects in Water. Technical Report, Naval Surface Weapons Center, Dahlgren, VA., 1978

[2] Daniel, I.M., Gdoutos, E., Rajapakse, Y.D. (eds.), Major Accomplishments in Composite Materials and Sandwich Structure: An Anthology of ONR Sponsored Research. Springer, 2010

[3] Nurick, G.N., Shave, G.C., The Deformation and Tearing of Thin Square Plates Subjected to Impulsive Loads-An Experimental Study. International Journal of Impact Engineering, 1996, 18(1), 99-116 
[4] Tilbrook, M.T., Deshpande, V.S., Fleck, N.A., Underwater Blast Loading of Sandwich Beams: Regimes of Behaviour. International Journal of Solids and Structures, 2009, 46(17), 3209-3221

[5] Latourte, F., Gregoire, D., Zenkert, D., Wei, X., Espinosa, H.D., Failure Mechanisms in Composite Panels Subjected to Underwater Impulsive Loads. Journal of the Mechanics and Physics of Solids, 2011, 59(8), $1623-1646$

[6] Carnell, M.T., Emmony, D.C., Quantitative Schlieren Measurements of a High-Energy Electromagnetic Transducer Acoustic Shock Field. Optics and Laser Technology , 1994, 26(6), 385-391

[7] Sommerfeld, M., Muller, H., Experimental and Numerical-Studies of Shock-Wave Focusing in Water. Experiments in Fluids, 1988, 6(3), 209-216

[8] Milton, B., Archer, R., Generation of Implosions by Area Change in a Shock Tube. AIAA Journal, 1969, 7(4), $779-780$

[9] Inoue, O., Takahashi, N., Takayama, K.: Shock Wave Focusing in a Log-spiral Duct. AIAA Journal, 1993, $31,1150-1152$

[10] Shepherd, J., Inaba, K., Shock Loading and Failure of Fluid-Filled Tubular Structures. (Dynamic failure of Materials and Structures Eds: Shukla A. etc.). Springer, 2010, 153-190

[11] Eliasson, V., Mello, M., Rosakis, A.J., Dimotakis, P.E., Experimental Investigation of Converging Shocks in Water with Various Confinement Materials. Shock Waves, 2010, 20(5), 395-408

[12] Anderson, W.W., Cherne, F.J., Zocher, M.A., Material Properties under Intensive Dynamic Loading. (Shock Wave and High Pressure Phenomena). Springer, 2010

[13] Whitham, G.B., Linear and Nonlinear Waves. Wiley-Interscience, 2011

[14] Inoue, O., Imuta, S., Milton, B., Takayama, K., Computational Study of Shock Wave Focusing in a Log-spiral Duct. Shock Waves, 1995, 5, 183-188

[15] Ridah, S., Shock Waves in Water, Journal of Applied Physics, 1988, 64, 152-158

[16] Cocchi, J., Saurel, R., Loraud, J., Treatment of Interface Problems with Godunov-type Schemes, Shock Waves, 1996, 5, 347-357.

[17] Johnsen, E, Colonius, T., Shock-induced Collapse of a Gas Bubble in Shockwave Lithotripsy, Journal of the Acoustical Society of America. 2008, 124, 2011-2020.

[18] Cole, R. H., Underwater explosions. Princeton University Press, 1948.

[19] Cooper, P., Explosives Engineering, Wiley-VCH, 1996

[20] Nagayama, K., Mori, Y., Shimada, K., Nakahara, M., Shock Hugoniot Compression Curve for Water up to 1 GPa by Using a Compressed Gas Gun. Journal of Applied Physics, 2002, 91, 476-482

[21] Millett, J., Bourne, N. K., Shock and Release of Polycarbonate under One-dimensional Strain. Journal of Material Science, 2006, 41, 1683-1690

[22] Settles, G.S., Schlieren and Shadowgraph Techniques. (Visualizing Phenomena in Transparent Media). Springer Verlag, 2001

[23] Matula, T. J., Marston, P. L., Energy Branching of a Subsonic Flexural Wave on a Plate at an Air-water interface. I. Observation of the Wave Field near the Interface and Near the Plate, Journal of the Acoustical Society of America. 1995, 97, 1389-1398

[24] Mouritz, A.P., Gellert, E., Burchill, P., Challis, K., Review of Advanced Composite Structures for Naval Ships and Submarines. Composite Structures, 2001, 53(1), 21-41

[25] Abrate, S., Impact on Laminated Composite Materials. Applied Mechanics Reviews, 1991, 44(4), 155-191

[26] Carlsson, L., Adams, D.F., Pipes, R.B., Experimental Characterization of Advanced Composite Materials, 3rd edn. CRC Press, 2002

[27] Kwon, Y., Multiscale and Multilevel Modeling of Composites. Springer, 2007

[28] Carruthers, J., Kettle, A., Robinson, A., Energy Absorption Capability and Crashworthiness of Composite Material Structures: A Review. Applied Mechanics Reviews, 1998, 51(10), 635-650

[29] Cantwell, W., Morton, J., The Impact Resistance of Composite Materials-A Review. Composites, 1991, 22(5), 347-362 
